\title{
Global gyrokinetic turbulence simulations of MAST plasmas
}

\author{
S. Saarelma ${ }^{1}$, P. Hill ${ }^{3}$, A. Bottino ${ }^{2}$, G. Colyer ${ }^{1}$, A. Field ${ }^{1}$, B. \\ McMillan ${ }^{3}$, A. Peeters ${ }^{4}$, C.M. Roach ${ }^{1}$ and MAST team ${ }^{1}$ \\ ${ }^{1}$ EURATOM/CCFE Fusion Association, Culham Science Centre, Abingdon, OX14 \\ 3DB, UK \\ ${ }^{2}$ MPI für Plasmaphysik, EURATOM Association, D-85748 Garching, Germany \\ ${ }^{3}$ Centre for Fusion, Space and Astrophysics, Warwick University, Coventry CV4 \\ $7 \mathrm{AL}, \mathrm{UK}$ \\ ${ }^{4}$ Department of Physics, University of Bayreuth, D-95440 Bayreuth, Germany
}

\begin{abstract}
Local and global electrostatic gyrokinetic simulations of MAST spherical tokamak for ITG modes with adiabatic electron response find good agreement in linear stability with growth rates below the experimental $\mathbf{E} \times \mathbf{B}$ shearing rate. The kinetic treatment of electrons is shown to increase the growth rates above the shearing rate. The collisionless simulations with kinetic electrons and experimental flow shear find unstable modes in the outer part of the plasma. In global simulations the flow shear stabilisation is found to be asymmetric with respect the direction of flow with a small destabilising effect with a modest flow in the co-direction.

The global non-linear simulations show turbulence spreading from the outer part of the plasma into the linearly stable core region. With adiabatic electrons and experimental profiles the turbulent heat transport is at or below the neoclassical level even without the flow shear. Treating electrons kinetically and including the flow shear effects increases the turbulent transport well above the neoclassical level.
\end{abstract}

PACS numbers: $52.55 . \mathrm{Fa}$

Submitted to: Plasma Physics and Controlled Fusion 


\section{Introduction}

The anomalous transport from ion scale microturbulence driven by ion temperature gradient (ITG) modes and trapped electron modes (TEM) in spherical tokamaks such as MAST and NSTX is thought to be suppressed by sheared $\mathbf{E} \times \mathbf{B}$ flow in most high confinement mode (H-mode) discharges leaving only the electron scale turbulence driven by the electron temperature gradient (ETG) modes. However, in low confinement mode (L-mode) plasmas ion scale turbulence can still play an important role in transport $[1,2,3]$.

Gyrokinetic simulations have long been used to simulate linear and non-linear behaviour of ITG modes [4]. Most studies have consentrated on large aspect ratio tokamaks where the ratio of ion gyroradius to plasma size $\left(\rho_{i *}\right)$ is small (for instance in the benchmark study by Dimits, $\rho_{i *}=1 / 160$ [5]). The local treatment of the turbulence is usually sufficient, since the turbulent eddie size created by microinstabilities generally scale with Larmor radius of the particles driving the instabilities [6]. However, non-local effects on ion scale turbulence are likely to be important in devices where is $\rho_{i *}$ large. Firstly the equilibrium profiles vary accross the radial extent of the turbulent eddies, and secondly turbulence spreading can become important $[7,8]$. While the local gyrokinetic flux-tube approximation provides guidance on the linear microstability of ITG and TEM turbulence, in non-linear simulations of ITG turbulence at large $\rho_{i *}$ a global treatment of the plasma is required for a more quantitative description of the turbulent transport processes.

In the MAST spherical tokamak (ST), where the toroidal magnetic field at the plasma centre $B \approx 0.5 \mathrm{~T}$ and the ion temperature can exceed $1 \mathrm{keV}$ in the core, the condition of large $\rho_{i *}$ is fulfilled. Another important difference to the large aspect ratio tokamaks is a large trapped particle fraction due to the strong variation of magnetic field inside the plasma. Furthermore, the core plasma in a spherical tokamak that is heated with neutral beams, can reach very high rotation velocities. On the other hand, the flow of plasma outside the separatrix is restricted. This produces a steep flow shear gradient through the plasma. The sheared flow can have a stabilising effect on the microinstabilities [10].

In this paper we will present linear microstability results for ITG and TEM from local and global gyrokinetic codes, and we will show global non-linear simulations of ITG and TEM turbulence in MAST. Furthermore we show how the flow shear affects the turbulence.

\section{Experimental plasma}

For the microstabilty analysis we choose a MAST L-mode discharge \#22807 at 0.25 $\mathrm{s}$ that displays a steep ion temperature gradient at the location where there is also a steep gradient in the toroidal rotation [9]. The core has a negative magnetic shear up to $s=0.55$ where $s$ is the square root of the normalised poloidal flux. The ion 

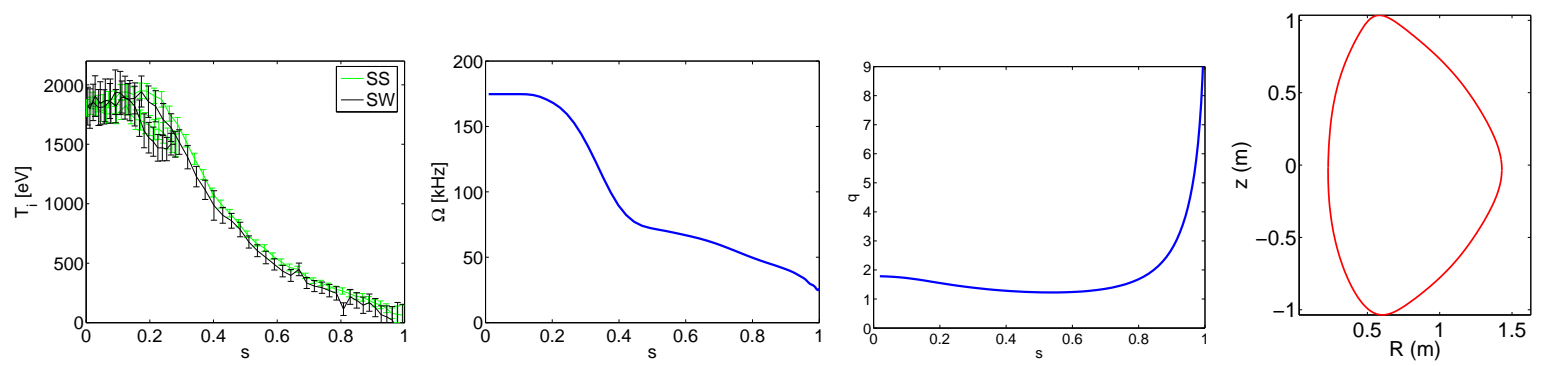

Figure 1. The ion temperature, toroidal rotation and q-profile and the plasma shape of the MAST discharge \#22807 at $0.25 \mathrm{~s}$. The two measurements of ion temperature refer to the two independent neutral beams used by the CXRS system. The inboard side measurements are folded onto the outboard side in $s<0.3$. The internal transport barrier (ITB) with steep $\nabla T_{i}$ is in the region $0.3<s<0.5$.

temperature profile from the charge exchange recombination spectroscopy (CXRS), the q-profile from MSE-constrained equilibrium reconstruction and the plasma shape are shown in Fig.1. The global parameters are: $I_{p}=0.88 \mathrm{MA}, B_{T}($ at $R=1 \mathrm{~m})=0.41 \mathrm{~T}$ and co-heating $P_{N B I}=3.3 \mathrm{MW}$. The steep ion temperature gradient coincides with a steep gradient in the toroidal rotation profile. In the outer part of the plasma, both the ion temperature gradient and the toroidal flow shear are significantly smaller than in the core. The value of $\rho_{i *}$ at $s=0.5$ is 0.018 , which indicates that global effects are likely to play a role in microstability studies, especially in the non-linear phase.

The experimental ion heat flux is determined from power balance using TRANSP code [11]. The neoclassical flux, the time variation of the equilibrium profiles and the collisional heat exchange between electrons and ions are subtracted from the heat sources and the residual is considered to be the anomalous heat flux. Unfortunately, even though the input neutral beam power is well known and it is possible to calculate the heat deposition from the fast ions based on particle orbits and collisions, the heat source distribution inside the plasma is sensitive to the so-called anomalous fast-ion diffusion. In addition to the neoclassical transport the fast ions from the neutral beam are transported from the core by the turbulence and MHD events. They are not modelled by TRANSP and have to be included by parameters (generally a global fast ion diffusion rate) given by the user. The common method is to adjust the fast ion diffusion rate so that the neutron rate from the fusion reactions matches with that of the experiment. However, this is a relatively crude method and leaves a significant uncertainty in the source term and, consequently, the calculated anomalous ion heat flux.

In this particular discharge, we find that the anomalous ion heat flux is relatively well determined before the second heating beam is turned on (flux variation between TRANSP time points is relatively small), but after that the variation between time points becomes so large that it is impossible to distinguish any finite anomalous flux from the noise. Therefore, we will use the anomalous ion heat flux from an earlier time point $(t=0.20 \mathrm{~s})$ in comparisons of the simulated flux with the experimental flux. 


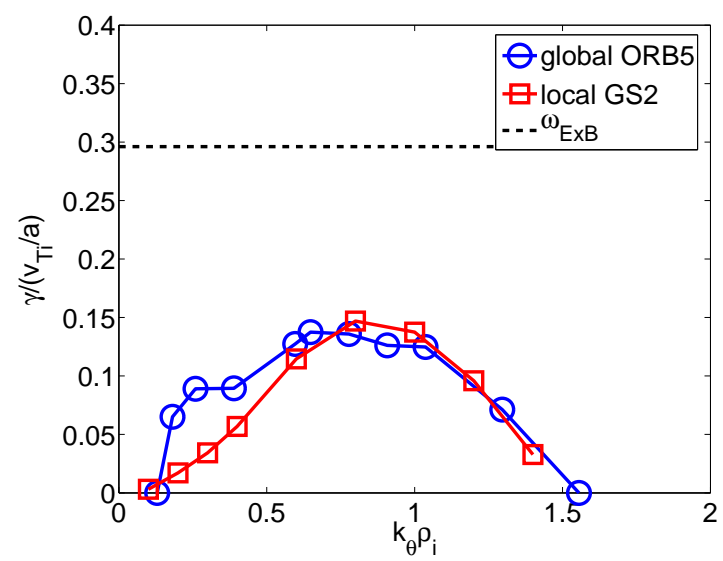

Figure 2. The linear spectrum of ITG mode growth rates from electrostatic simulation with adiabatic electron response using local flux tube code GS2 (green) and global code ORB5 (blue).

\section{Linear Gyrokinetic Simulations}

To study the linear plasma microstability, we use two different electrostatic gyrokinetic codes. For local analysis we use the flux tube code GS2 [13] and compare the local results with the results from the global code ORB5 [14]. While the non-local effects play can play a significant role in the non-linear phase of the turbulence simulation, in the linear phase a flux tube approach turns out to be sufficient.

From a global linear run of ORB5, we can determine the most unstable part of the plasma, i.e. where the mode starts to grow first. We accompany this simulation with simulations using an annular plasma that does not include the most unstable part to determine if other regions are unstable. We find that even though the maximum of $\nabla T_{i} / T_{i}$ is in the core region of the plasma $(s<0.5)$, the core is stable against ITG modes. In the core the magnetic shear $\hat{s}$ is reversed, which can explain the good stability [19]. The most unstable region in this plasma is between $s=0.6$ and $s=0.8$.

In Figure 2 the linear growth rate spectrum of the global ORB5 ITG simulation with adiabatic electron response is compared with a local GS2 flux tube simulation at $s=0.73$. From the good agreement we can see that for a linear analysis, the flux tube approach is sufficient.

To get further insight into the question of validity of local simulation, we vary $\rho^{*}$ in ORB5 simulations and find that the growth rate varies significantly for values above the experimental $\rho^{*}$, but below that the global simulation saturates. Figure 3 shows how the linear growth rate of the most unstable mode varies with the $\rho_{*}$ parameter. The limit where the global effects start to affect the linear growrth rate are just below the experimental value of $\rho_{*}$ of this MAST discharge. This agrees well with the result obtained by Lin for large aspect ratio tokamak [17].

The equilibrium flow shear has been included in ORB5 with a background radial 


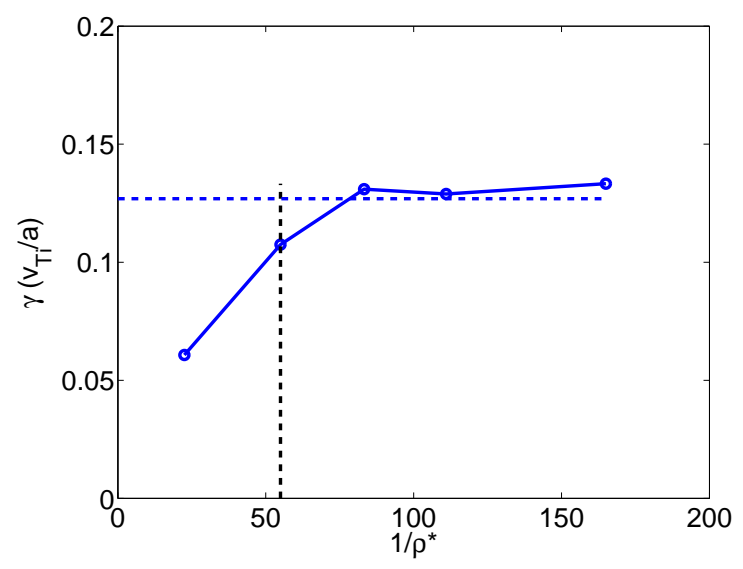

Figure 3. The growth rate of $k_{\theta} \rho_{i}=0.65$ mode as a function of $\rho_{*}$. The vertical line represents the experimental value. The horizontal line represents the local flux tube result obtained using GS2.

electric field and a shifted Maxwellian distribution function for the equilibrium quantity $f_{0}$ :

$$
f_{0}=\frac{n_{0}\left(\psi_{c}\right)}{\left(2 \pi T\left(\psi_{c}\right) / m\right)^{3 / 2}} \exp \left(-\frac{\epsilon-q \phi_{0}\left(\psi_{*}\right)}{T\left(\psi_{c}\right)}\right),
$$

where $m, q, T$ and $n_{0}$ are the mass, charge, temperature and density, $\epsilon=m v^{2} / 2+q \phi_{0}(\psi)$ is the energy, $\psi$ is the poloidal magnetic flux, $\psi_{c}$ is the canonical momentum and $\psi_{*}$ is the modified canonical momentum that is used to vary the flow from pure toloidal to pure poloidal flow [15].

Using the "pure toroidal flow" canonical momentum $\left(\psi_{*}=\psi(\vec{R})+F v_{\|} B\right.$, where $F$ is the poloidal current flux function) and the experimental plasma, we analyse the stabilising effect of the sheared flow on the linear ITG modes. The above formulation is valid for low flow shears, i.e. $q E_{r} \rho_{i}(a / R q)^{1 / 2} \ll T_{i}$. In the investigated MAST plasma this condition is satisfied everywhere except the very edge $(s>0.9)$. Since we are not trying to simulate the edge turbulence in any case, this is not a concern.

As shown in Fig. 4, the stabilising effect is asymmetrical with respect of the direction of the flow shear. If the flow profile is monotonic and the flow is in the co-direction (flow and plasma current are in the same direction, $d v_{\phi} / d s<0$ ), a small amplitude toroidal flow actually destabilises the ITG mode. In the counter-direction, any finite flow is stabilising. The physical explanation for the asymmetry is given by Kishimoto [18]. Depending on the relative signs of the shear in the eigenfrequency of the drift wave and the shear in the toroidal rotation, the a small flow shear either compensates the mode tilting caused by the diamagnetic shear thus destabilising the mode, or it enhances the tilting and stabilises the mode. A large flow shear dominates the shear in the eigenfrequency and is always stabilising.

We have found that the required $\mathbf{E} \times \mathbf{B}$ shearing rate to stabilise the ITG modes in MAST is 4 times the growth rate of the mode. The most unstable modes are stabilised 


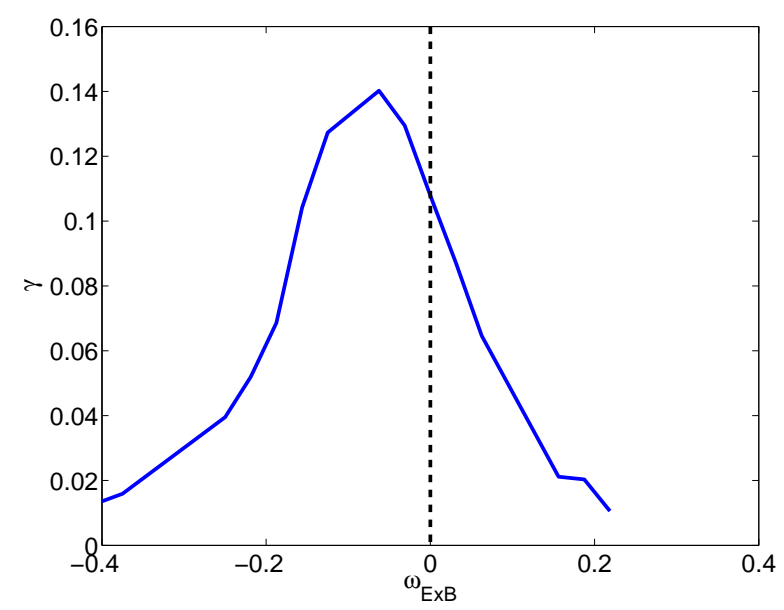

Figure 4. The growth rate of $k_{\theta} \rho_{i}=0.9$ ITG mode as a function of the toroidal flow. The negative values represent core rotation in the co-direction with the plasma current and positive values counter-direction.

a)

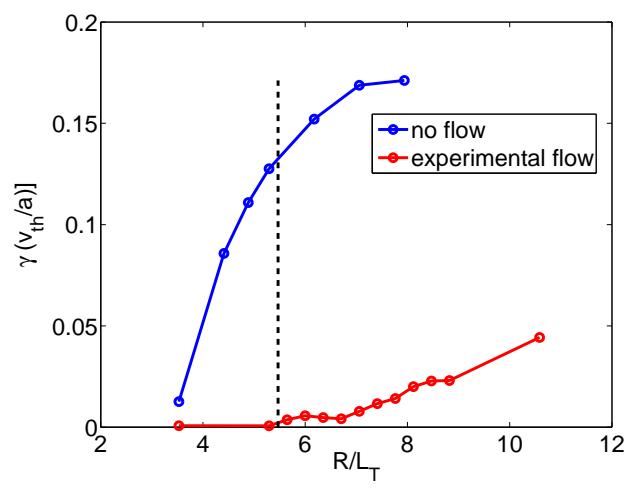

b)

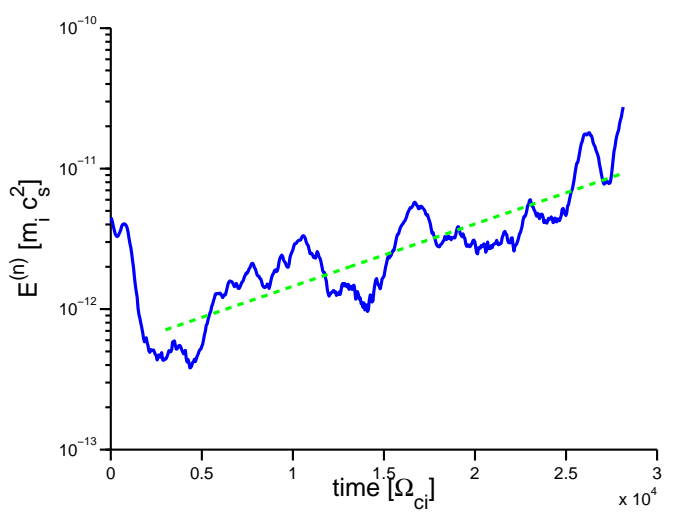

Figure 5. a) The growth rate of the most unstable ITG mode as a function of normalised ion temperature gradient $R / L_{T}$ for ORB5 simulations with and without the experimental flow. The vertical line shows the experimental ion temperature gradient. The growth rates with flow are averaged over fluctuations as shown shown in b).

more effectively than those with smaller growth rates making the spectrum almost flat at the experimental level of flow shear. It must be noted that all the growth rates at the experimental level of flow shear are very small $<0.01 v_{T i} / a$. Figure 5 shows how the growth rates with and without experimental flow vary as a function of the ion temperature gradient. While without the flow shear effects the plasma is well unstable against the ITG even below the experimental ion temperature gradient, including the effects puts the experimental plasma just at the marginal stability against the ITG modes.

Since the trapping fraction is roughly proportional to the square root of the inverse aspect ratio, in a spherical tokamak there are far more trapped particles than in a large 


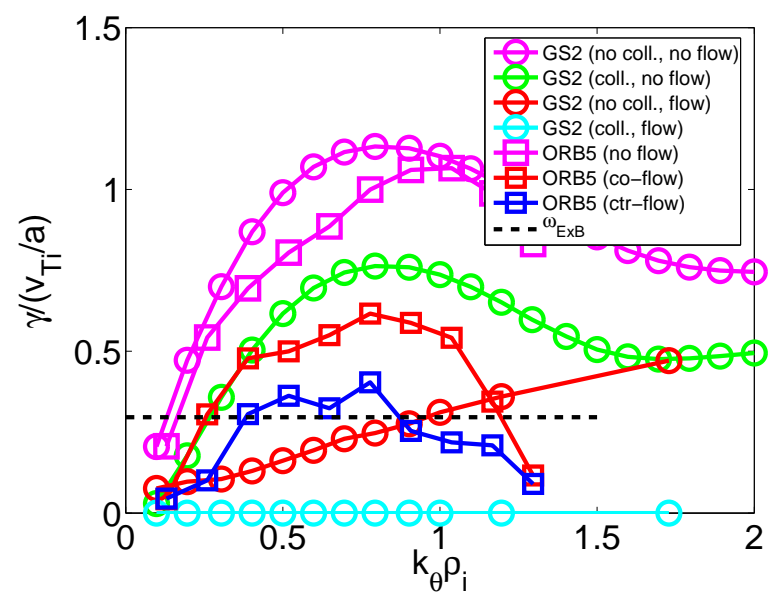

Figure 6. The linear spectrum of growth rates from simulation with kinetic trapped electrons using local flux tube code GS2 (circles) with no flow and no collisions (magenta), no flow and collisions (green), experimental flow and no collisions (red) and experimental flow and collisions (cyan) and global code ORB5 without collisions (squares) and no flow (magenta), experimental flow in the co-direction (red) and experimental flow in the counter-direction (blue).

aspect tokamak. Even though for low- $k_{\perp}$ modes the passing electrons can be assumed to respond adiabatically to the perturbed electric field, the kinetic treatment of trapped electrons can increase the drive of the ITG modes and can drive trapped electron modes $[8,16]$.

Figure 6 shows how the linear growth rate of ion scale turbulence increases when the trapped electrons are included kinetically. The collisionless global ORB5 result follows well the collisionless GS2 flux tube result. Including the collisions in the GS2 decreases the growth rates, but they still exceed the ITG growth rates with adiabatic electrons and also the experimental flow shear rate. We also find that the growth rates are not sensitive to the ratio $m_{e} / m_{i}$ used in the simulation. The change in the linear growth rate is less than $10 \%$ when $m_{e} / m_{i}$ is varied 3 orders of magnitude from the physical value. Therefore, in the ORB5 simulations we use increased ratio of $m_{e} / m_{i}$ of 0.01 instead of real value of 0.00054 for deuterium plasma in the non-linear simulations to save computing resources.

Including the flow shear effects, we find again the asymmetry in the global code ORB5 with respect to the flow direction. However, unlike in the case with adiabatic electron response, with kinetic trapped electrons the growth rates stay well above the zero when the experimental flow shear is taken into account. This is true also in local GS2 simulation. However, the effect on the unstable spectrum is quite different. In GS2 the long wave length modes are stabilised more effectively and in ORB5 the short wave length modes. If both collisions and the flow shear effects are taken into account in GS2, the entire spectrum becomes stable. 


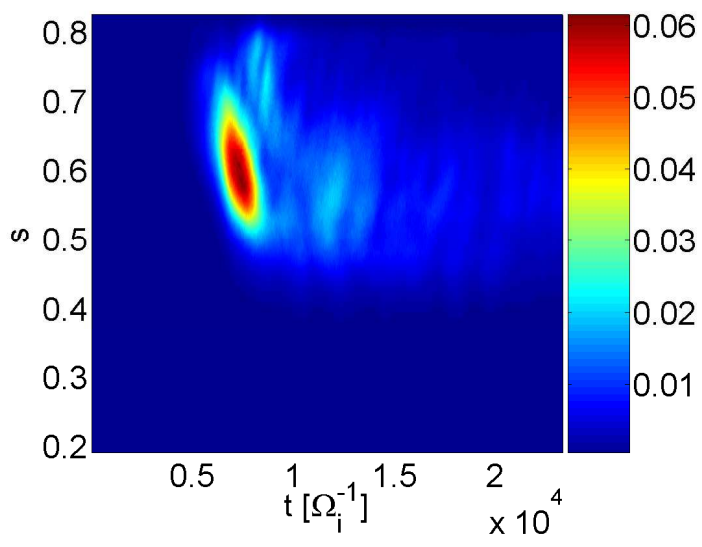

Figure 7. The ion heat flux (in $\mathrm{MW} / \mathrm{m}^{2}$ ) as a function of $s$ and time in a ORB5 simulation with experimental equilibrium and profiles and adiabatic electron response.

\section{Global Non-linear Gyrokinetic Simulations}

While the linear analysis can be used to study the stability of the plasma, only nonlinear simulations can be used to estimate the tranport rate of energy and particles that the turbulence produces. ORB5 avoids the problem of noise by using a filter to eliminate unphysical modes, i.e. modes that do not have $m / n \approx q$ as well as a relaxation operator that ensures that the marker weights stay bounded but does not affect the zonal flow dynamics $[14,22]$. The heating operator is used to keep temperature profiles from relaxing during the simulation. The heating operator does not try to simulate the experimental heating profile, but only pushes the perturbed profiles towards the initial equilibrium profiles.

\subsection{Adiabatic Electrons}

The non-linear ORB5 simulations are done using $2 \times 10^{8}$ markers on a $N_{s} \times N_{\chi} \times N_{\phi}=$ $50 \times 512 \times 256$ grid ( $s$ is the radial, $\chi$ is the perpendicular and $\phi$ is the toroidal coordinate) Using the adiabatic response, i.e. no trapped electron drive, produces turbulence that spreads from the linearly unstable region to the linearly stable region during the nonlinear phase. This can be seen in Fig. 7 where the ion heat flux is plotted as a function of time and radius. The turbulence starts around $s \approx 0.7$ (which was found to be the most unstable region linearly), but then spreads inwards all the way to $s \approx 0.4$. During the saturated phase the the maximum amplitude of the heat flux is located between $s=0.4$ and $s=0.7$. The signal to noise ratio stays above 20 through the simulation. For the grid convergence test, we did runs with twice the radial points. The fluxes and non-linear spectrum are unaffected, but the signal to noise ratio is halved.

When we compare the level of turbulent transport created by saturated ITG turbulence, we find that at experimental temperature profiles and even without 


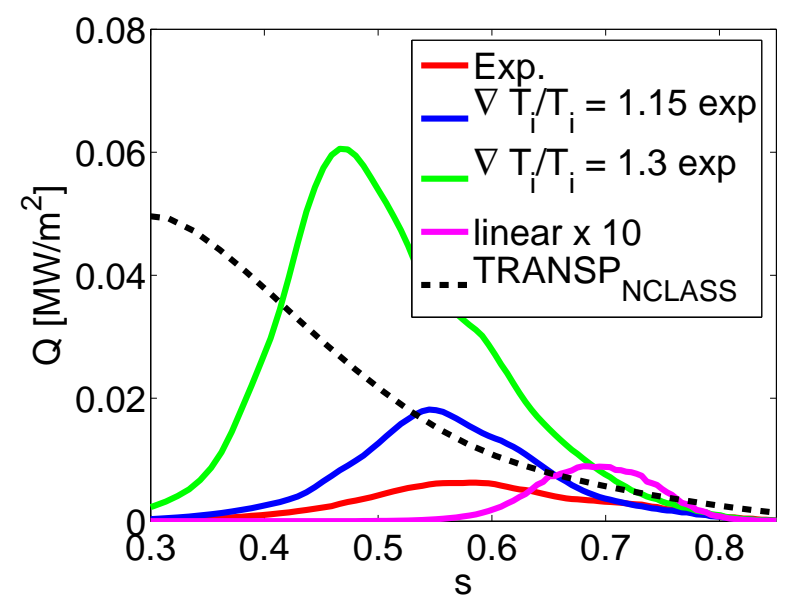

Figure 8. The ion heat flux as a function of $s$ in a ORB5 simulation with experimental (red), $15 \%$ increased (blue) and $30 \%$ increased ion temperature gradients. Also plotted is the neoclassical heat flux calculated by TRANSP (dashed line) as well as the region where the mode grows in the linear phase (magenta).

considering any equilibrium flow shear stabilisation, the turbulent heat flux is very low even below the neolassical level calculated by NCLASS in TRANSP (Fig. 8). Increasing the ion temperature gradient in the core region $(s=0.3-0.5)$ by $30 \%$ increases the heat flux well above the neoclassical level. It would seem that in the steep pressure gradient region the plasma ion temperature profile is just below the non-linear threshold for ITG.

\subsection{Kinetic Trapped Electrons}

We include the trapped electrons kinetically into the simulation, but use the mass ratio $m_{e} / m_{i}=0.01$ instead of the real value in order to avoid making the time step too short. As was shown in the previous section, the linear growth rate becomes much larger when the trapped electrons are included. It also leads to significantly higher ion heat flux in the non-linear phase. Using the experimental profiles and assuming no sheared flow, the ion heat flux is well above both the neoclassical heat flux and the experimental level of anomalous heat flux calculated by TRANSP (Figure 9). As in the case of ITG with adiabatic electrons, also in this case the turbulence spreads from the linearly unstable region (which in this case is even further from the core than in the ITG simulation) towards the core and actually the maximum heat flux during the saturated turbulence is in the linearly stable region where the magnetic shear is negative (Fig. 10).

In the outer region $(s>0.5)$ the turbulent heat flux with flow shear effects taken into account is well above both the neoclassical level and the anomalous level calculated by TRANSP. However, unlike without flow shear the spread of turbulence is stopped into the steep flow shear region $(s<0.5)$. It is also interesting to note that while there is an asymmetry in linear growth rates with respect to the direction of the flow, the non-linear heat flux shows no indication of such asymmetry. We have shown that the 


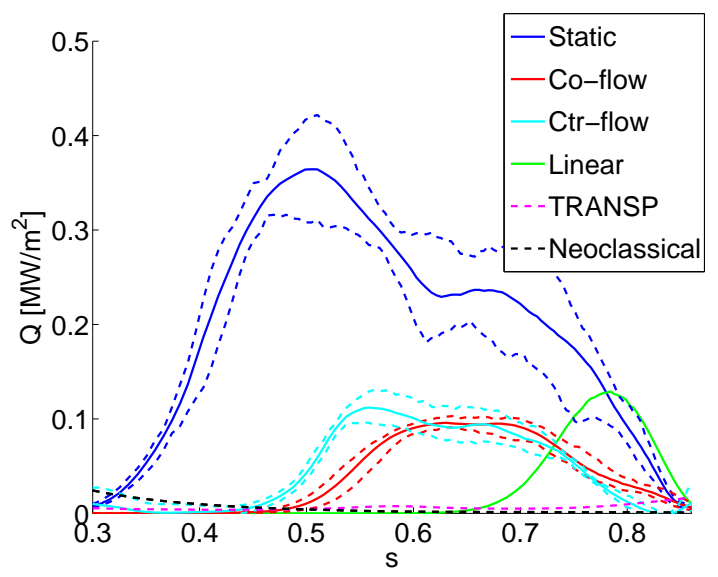

Figure 9. The ion heat flux in a ORB5 simulation with kinetic trapped electrons with co-flow (red), counter-flow (cyan) and without flow (blue) as a function of $s$ compared with the experimental anomalous (magenta) and neoclassical (black) heat flux calculated by TRANSP. The dashed blue, red and cyan lines show the variation of the simulated flux during the saturated non-linear phase. The green curve shows the location of the instability during the linear phase.
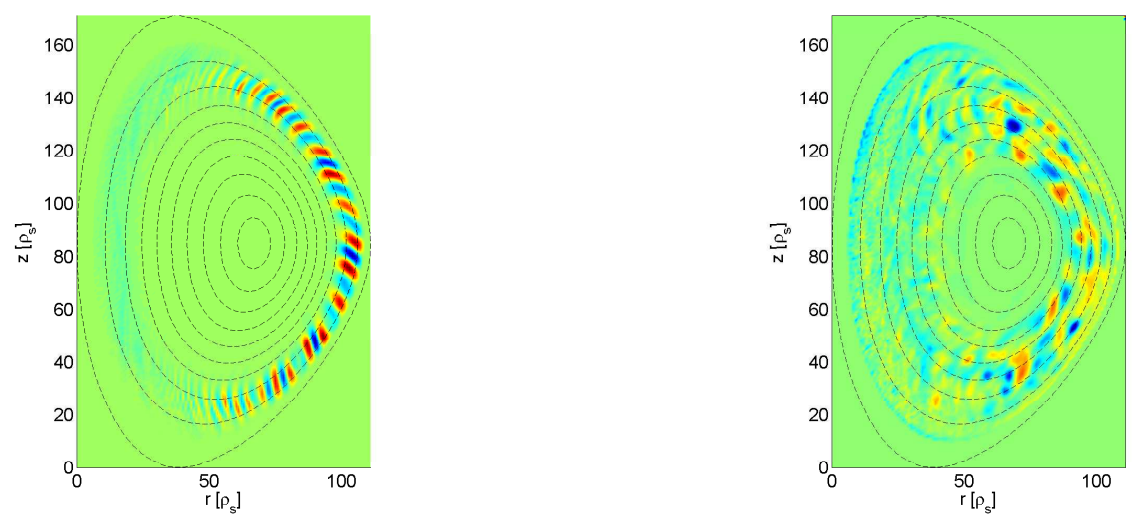

Figure 10. The perturbed potential $\phi-\langle\phi\rangle$ (where $\langle\phi\rangle$ is the flux surface averaged potential) during the linear (left) and fully saturated (right) phase of the ORB5 simulation where trapped electrons were treated kinetically.

collisions will decrease the trapped electron drive, and, therefore, is likely to decrease the non-linear flux as well in the outer region as well. The quantification of this effect will be left for future work.

During the non-linear phase the energy spectrum shifts towards long wave length modes from the linearly most unstable modes. As shown in Fig. 11 most of the energy in the non-linear phase is with the modes that have $k_{\theta} \rho_{i} \approx 0.3$, about half of the linear growth rate spectrum peak. In the non-linear phase the zonal flows play an important role regulating the turbulence. Their effect on the spectrum is similar to the equilibrium flow shear's effect on linear modes. The short wave length modes are stabilised more than the long wave length modes. The increased size of the turbulent eddies in the 


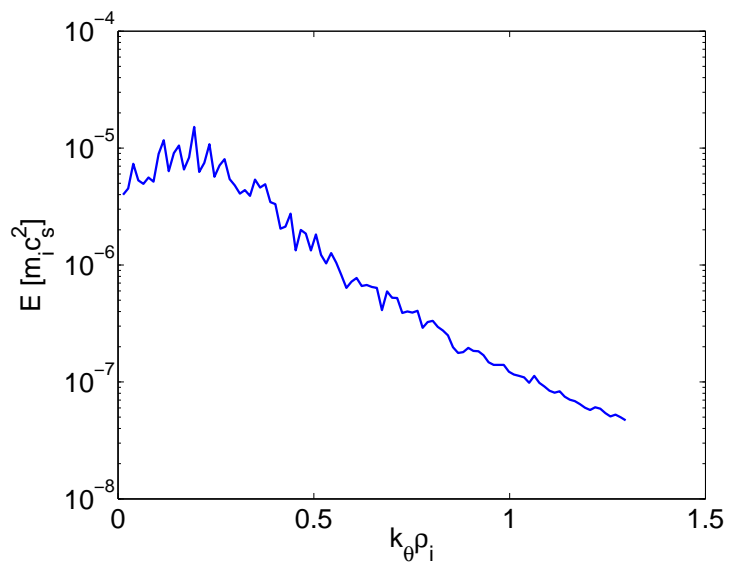

Figure 11. The non-linear spectrum for the ORB5 simulation including the kinetic trapped electrons.

non-linear phase can also be seen in Fig. 10 where the perturbed potential is plotted in a poloidal cross section of the plasma during the linear and non-linear phase.

\section{Conclusions}

The gyrokinetic modelling of ion scale turbulence in MAST L-mode plasma with a $\rho_{*}=0.018$ shows clear non-local behaviour. While the linear unstable spectrum agrees well with the local flux tube analysis, the non-linear spreading of turbulence can only be observed in a global simulation. In global gyrokinetic simulations we find non-linear spreading of turbulence from the linearly unstable outer region of the MAST plasma into the linearly stable core. In the saturated non-linear turbulent phase the maximum of the heat flux is in the linearly stable core region.

The flow shear has an asymmetric effect on the ITG stabilisation with respect to the direction of the flow. The flow in the co-direction is destabilising at low flow shear, but stabilising at high flow shear. The counter-flow is stabilising for all flow shearing rates. At the experimental level of flow shear, the MAST plasma is just at the linear stability boundary for ITG modes when electrons are treated adiabatically. Treating trapped electrons kinetically in the simulations increases the growth rates and results in unstable modes even when experimental flow shear is taken into account. Additionally, the flow shear moves the unstable mode spectrum towards long wave length modes by stabilising the short wave length modes.

The level of turbulent heat flux from ITG modes with adiabatic electron response is very small for the experimental profiles, but increases well above the neoclassical level with a modest increase of the temperature gradient. Including the trapped electron physics, both the linear growth rates and especially the non-linear heat flux increase significantly. The flow shear reduces the heat flux, but the level of turbulent flux remains well above the neoclassical level when the trapped electrons are treated kinetically in 
the simulations.

\section{Acknowledgments}

This work, part-funded by the European Communities under the contract of Association between EURATOM and CCFE, was carried out within the framework of the European Fusion Development Agreement. The views and opinions expressed herein do not necessarily reflect those of the European Commission. The computations were done using HECToR (grant number EP/H002081/1) and HPC-FF supercomputers.

\section{References}

[1] S M Kaye et al., Nucl. Fusion 47 (2007) 499

[2] C M Roach et al, Plasma Phys. Control. Fusion 47 (2005) B323

[3] R J Akers et al. "Transport studies in the MAST spherical tokamak", Proc. 22nd IAEA Fusion Energy Conf. (Geneva, Switzerland, 2008) EX2-2

[4] S E Parker et al., Phys. Rev. Lett. 71 (1993) 2042

[5] A M Dimits et al., Phys. Plasmas 7 (2000) 969

[6] J Candy, R E Waltz and W Dorland, Phys. Plasmas 5 (2004) L25

[7] Z Lin and T S Hahm, Phys. Plasmas 11 (2004) 1099

[8] R D Sydora, V K Decyk, and J M Dawson, Plasma Phys. Controlled Fusion 38 (1996) A281

[9] A R Field et al. "Plasma Rotation and Transport in the MAST Spherical Tokamak", Proc. 23rd IAEA Fusion Energy Conf. (Daejon, Korea, 2010) EXC/P8-04, Accepted for publication in Nucl. Fusion

[10] A R Field et al, "Core heat transport in the MAST spherical tokamak" Proc. 20th IAEA Fusion Energy Conf. (Vilamoura, Portugal, 2004) EX/P2-11

[11] R J Goldston et al J. Comput. Phys. 43 (1981) 61

[12] K H Burrell et al., Phys. Plasmas 6 (1999) 4418

[13] M Kotschenreuther, G Rewoldt and W M Tang Comput. Phys. Commun 88 (1995) 128

[14] S Jolliet, et al. Comput. Phys. Commun. 177 (2008) 409

[15] B F McMillan, et al., "Effects of toroidal and poloidal shear flow in global gyrokinetic simulations" 37th EPS Conference on Plasma Physics, Dublin, 21-25 June 2010, P1.1063

[16] T Dannert and F Jenko Phys. Plasmas 12 (2005) 072309

[17] Z Lin, S Ethier, T S Hahm and W M Tang, Phys. Rev. Lett. 88 (2002) 195004

[18] Y Kishimoto et al., Plasma Phys. Control. Fusion 41 (1999) A663

[19] J F Drake et al, Phys. Rev. Lett. 77 (1996) 494

[20] R E Waltz, G D Kerbel and J Milovich, Phys. Plasmas 1 (1994) 2229

[21] C M Roach, et al., Plasma Phys Contr. Fusion 51 (2009) 124020

[22] B F McMillan, et al., Phys. Plasmas 15 (2008) 052308x 